\title{
SUR LA LINÉARITÉ DE LA FONCTION DE ARTIN
}

\author{
PAR GUILlAUME ROND
}

RÉSUMÉ. - Nous donnons ici un contre-exemple à une vieille conjecture en théorie des singularités. Cette conjecture est que la fonction qui apparaît dans la version forte du théorème d'approximation de Artin est majorée par une fonction affine. Tout d'abord nous faisons une étude de l'approximation diophantienne entre le corps des séries en plusieurs variables et son complété pour la topologie m-adique. Nous montrons, à l'aide d'un exemple, qu'il n'existe pas de version du théorème de Liouville dans ce contexte. Ce même exemple nous fournit notre contre-exemple (théorème 1.2). Nous appliquons cela pour donner une nouvelle preuve du fait qu'il n'existe pas de théorie de l'élimination des quantificateurs pour le corps des séries en plusieurs variables.

(C) 2005 Elsevier SAS

ABSTRACT. - We give here a counter-example to an old conjecture in the theory of singularities. This conjecture is that the function that appears in the strong Artin approximation theorem is bounded by an affine function. First we study Diophantine approximation between the field of power series in several variables and its completion for the $\mathfrak{m}$-adic topology. We show, with an example, that there is no Liouville theorem in this case. This example gives us our counter-example (cf. théorème 1.2). As an application, we give a new proof of the fact that there is no theory of elimination of quantifiers for the field of fractions of the ring of power series in several variables.

(c) 2005 Elsevier SAS

\section{Introduction}

En 1969, M. Artin a prouvé le théorème suivant (version forte du théorème d'approximation de Artin) :

THÉORÈME 1.1 [1]. - Soit I un idéal de $\mathcal{O}_{N}\left[X_{1}, \ldots, X_{n}\right]$ engendrépar les $f_{l}$ pour $1 \leqslant l \leqslant r$. Il existe une fonction $\beta: \mathbb{N} \rightarrow \mathbb{N}$ telle que :

$$
\begin{gathered}
\forall i \in \mathbb{N} \forall x \in \mathcal{O}_{N}^{n} \quad \text { tels que } \\
\left(\forall l f_{l}(x) \in \mathfrak{m}_{N}^{\beta(i)+1} \mathcal{O}_{N}^{r}\right) \Longrightarrow\left(\exists \bar{x} \in \mathcal{O}_{N}^{n} \text { tel que } \bar{x}-x \in \mathfrak{m}_{N}^{i+1} \mathcal{O}_{N}^{n} \text { et } \forall l f_{l}(\bar{x})=0\right)
\end{gathered}
$$

où $\mathfrak{m}_{N}$ est l'idéal maximal de $\mathcal{O}_{N}$, l'anneau des séries formelles en $N$ variables sur un corps $\mathbb{k}$ quelconque.

Pour $I$ un idéal de $\mathcal{O}_{N}\left[X_{1}, \ldots, X_{n}\right]$, nous appellerons fonction de Artin de $I$ la plus petite fonction $\beta: \mathbb{N} \rightarrow \mathbb{N}$ qui vérifie le théorème ci-dessus. Ce théorème nous dit donc que les solutions approchées des $f_{l}$ sont proches de vraies solutions pour la topologie $\mathfrak{m}$-adique, la fonction $\beta$ étant une «mesure» de cette approximation. Dans le cas $N=1$, M.J. Greenberg [5] a montré que la fonction de Artin d'un idéal était majorée par une fonction affine. Le fait que dans ce cas 
la fonction de Artin d'un idéal soit bornée par une fonction affine peut s'interpréter en terme d'inégalité de type Łojasiewicz sur l'espace des arcs (cf. remarque 2).

D'autre part, si $\mathcal{O}_{N} \rightarrow \mathcal{O}_{N}\left[X_{1}, \ldots, X_{n}\right] / I$ est lisse et $N$ quelconque, alors la fonction de Artin de $I$ est égale à l'identité. La fonction de Artin d'un tel idéal peut donc être vue comme une mesure de la lissité du morphisme précédent.

La conjecture suivante a été formulée en 1989 [13] (voir aussi [2]) :

CONJECTURE. - Toute fonction de Artin est bornée par une fonction affine.

Ce problème a été étudié, entre autres, par D. Delfino et I. Swanson, M. Hickel, S. Izumi, M. Spivakovsky, B. Teissier (cf. par exemple [8], [13], ou [2]).

Voici quelques exemples d'idéaux dont la fonction de Artin est bornée par une fonction affine (voir aussi [12]) :

- les idéaux principaux de $\mathcal{O}_{N}\left[X, Y, Z_{1}, \ldots, Z_{n}\right]$ engendrés par les polynômes de la forme $X Y-\sum_{i} f_{i} Z_{i}$ où l'idéal $\left(f_{1}, \ldots, f_{n}\right)$ est premier (théorème d'Izumi [8]),

- les idéaux de $\mathcal{O}_{N}\left[X_{1}, \ldots, X_{n}\right]$ engendrés par des polynômes homogènes de degré 1 (voir par exemple [12, théorème 3.1]),

- les idéaux principaux de $\mathcal{O}_{N}\left[X, X_{1}, \ldots, X_{n}\right]$ engendrés par les polynômes de la forme $X^{n}+f X^{n-1} X_{1}+\cdots+f^{n} X_{n}$ où $f \in \mathcal{O}_{N}$ [2, théorème 3.10].

Enfin, précisons que pour $N=1$, cette fonction apparaît dans l'étude de l'espace des arcs d'un germe singulier de variété [9] et en intégration motivique [4] (J. Denef et F. Loeser relient cette fonction avec certaines séries de Poincaré motiviques, cf. théorème 8.1).

Nous donnons ici un contre-exemple à cette conjecture :

THÉORÈME 1.2. - La fonction de Artin du polynôme

$$
P(X, Y, Z):=X^{2}-Z Y^{2} \in \mathcal{O}_{N}[X, Y, Z]
$$

est bornée inférieurement par une fonction polynomiale de degré 2 si $N \geqslant 2$ et si la caractéristique de $\mathbb{k}$ est différente de 2.

Nous relions tout d'abord un résultat de type approximation diophantienne sur le corps $\mathbb{K}_{N}$ (le corps des fractions de $\mathcal{O}_{N}$ ) à l'existence d'une fonction de Artin (proposition 2.1 et corollaire 2.3). Nous montrons ensuite, à l'aide d'un exemple (cf. exemple 2.4), qu'il n'existe pas de résultat du type théorème de Liouville pour une extension finie de $\mathbb{K}_{N}$ dans $\widehat{\mathbb{K}}_{N}$, son complété pour la topologie $\mathfrak{m}_{N}$-adique. Nous nous appuyons alors sur cet exemple pour donner notre contre-exemple à la conjecture citée précédemment (théorème 1.2). La preuve du théorème 1.2 (partie 3) peut se lire indépendamment de la partie 2 qui traite de l'approximation diophantienne. La partie 2 permet cependant d'éclairer le théorème 1.2.

Enfin, nous déduisons de cela, en dernière partie, qu'il n'existe pas de théorie d'élimination des quantificateurs dans $\mathbb{K}_{N}$, pour $N \geqslant 2$ (théorème 4.1 ), pour le langage défini dans la dernière partie.

Soient $N$ un entier positif non nul et $\mathbb{k}$ un corps ; nous utiliserons les notations suivantes :

- $\mathcal{O}_{N}$ est l'anneau des séries formelles $\mathbb{k}\left[\left[T_{1}, \ldots, T_{N}\right]\right]$ et $\mathfrak{m}_{N}$ son idéal maximal (ou $\mathfrak{m}$ quand il n'y aura pas d'équivoque possible sur $N$ ).

- ord est la valuation $\mathfrak{m}_{N}$-adique sur $\mathcal{O}_{N}$. Cette valuation définit une norme || $\operatorname{sur} \mathcal{O}_{N}$ en posant $|x|=e^{-\operatorname{ord}(x)}$ et cette norme induit une topologie appelée topologie $\mathfrak{m}$-adique.

- $V_{N}:=\left\{\frac{x}{y} \mid x, y \in \mathcal{O}_{N}\right.$ et $\left.\operatorname{ord}(x) \geqslant \operatorname{ord}(y)\right\}$, l'anneau de valuation discrète qui domine $\mathcal{O}_{N}$ pour ord. Nous noterons $\mathfrak{m}_{N}^{\prime}$ son idéal maximal.

- $\widehat{V}_{N}:=\mathbb{k}\left(\frac{T_{1}}{T_{N}}, \ldots, \frac{T_{N-1}}{T_{N}}\right)\left[\left[T_{N}\right]\right]$ est le complété pour la topologie $\mathfrak{m}_{N}^{\prime}$-adique de $V_{N}$. En effet, cet anneau correspond au complété le long du diviseur exceptionnel de l'anneau $\mathcal{O}_{N}$.

$4^{\text {e }}$ SÉRIE - TOME $38-2005-\mathrm{N}^{\circ} 6$ 
Nous noterons $\widehat{\mathfrak{m}}_{N}$ l'idéal maximal de cet anneau, ord l'extension de la valuation $\mathfrak{m}_{N^{-}}^{\prime}$ adique et || l'extension de la norme associée.

- $\mathbb{K}_{N}$ et $\widehat{\mathbb{K}}_{N}$ sont respectivement les corps de fractions de $\mathcal{O}_{N}$ et de $\widehat{V}_{N}$. On peut remarquer que $\widehat{\mathbb{K}}_{N}$ est le complété de $\mathbb{K}_{N}$ pour la norme ||.

Remarque 1. - Un théorème de Wavrik (cf. [14]) donne l'existence de la fonction de Artin pour les systèmes d'équations polynomiales à coefficients dans $\mathbb{k}\left\{T_{1}, \ldots, T_{N}\right\}$, l'anneau des séries convergentes, quand $\mathbb{k}$ est muni d'une norme. Tout ce qui est énoncé dans la suite est encore valable dans ce cas.

Remarque 2. - Soit $I=\left(f_{1}, \ldots, f_{p}\right)$ un idéal de $\mathcal{O}_{N}\left[X_{1}, \ldots, X_{n}\right]$. Notons $F$ le sousensemble de $\mathcal{O}_{N}^{n}$ défini par $f_{1}=\cdots=f_{p}=0$. Notons $d$ la distance sur $\mathcal{O}_{N}^{n}$ induite par la norme ||$_{\infty} \operatorname{sur} \mathcal{O}_{N}^{n}$, elle-même définie par $\left|\left(x_{1}, \ldots, x_{n}\right)\right|_{\infty}=\max _{i}\left|x_{i}\right|$. Alors la fonction de Artin de $I$ est bornée par une fonction affine si et seulement si nous avons l'inégalité suivante de type Łojasiewicz:

$$
\max _{i}\left|f_{i}\left(x_{1}, \ldots, x_{n}\right)\right| \geqslant K d(x, F)^{a}
$$

où $K$ et $a$ sont des constantes indépendantes des $x_{i}$ (pour une preuve de ce résultat, voir [7]).

\section{Polynôme homogène à zéro isolé et approximation diophantienne}

Nous allons montrer ici que la fonction de Artin d'un polynôme homogène à zéro isolé est bornée par une fonction affine si et seulement si nous avons un résultat de type approximation diophantienne (cf. remarque 3) :

Proposition 2.1. - Soit $P \in \mathcal{O}_{N}\left[X_{1}, \ldots, X_{n}\right]$ un polynôme homogène de degré d qui a pour unique zéro dans $\mathcal{O}_{N}^{n}$ le point $(0, \ldots, 0)$. Notons $Q_{i}\left(X_{1}, \ldots, \widehat{X}_{i}, \ldots, X_{n}\right)$ le polynôme $P\left(X_{1}, \ldots, 1, \ldots, X_{n}\right)$ où la variable $X_{i}$ est remplacée par 1 dans $P$, pour $i \in\{1, \ldots, n\}$.

Le polynôme $P$ admet une fonction de Artin bornée par une fonction affine si et seulement si il existe deux constantes positives a et $b$ telles que nous ayons

$$
\min _{j}\left\{\operatorname{ord}\left(\frac{u_{j}}{v}-y_{j}\right)\right\} \leqslant a \operatorname{ord}(v)+b
$$

pour tout $i \in\{1, \ldots, n\}$, pour toute racine $\left(y_{1}, \ldots, \widehat{y}_{i}, \ldots, y_{n}\right)$ de $Q_{i}$ dans $\widehat{V}_{N}$ et pour tout $u_{1}, \ldots, \widehat{u}_{i}, \ldots, u_{n}$ et $v$ dans $\mathcal{O}_{N}$.

Remarque 3. - La condition (1) peut aussi s'énoncer sous la forme suivante : il existe deux constantes positives $c$ et $K$ telles que pour tout entier $i \in\{1, \ldots, n\}$, pour toute racine $\left(y_{1}, \ldots, \widehat{y}_{i}, \ldots, y_{n}\right)$ de $Q_{i}$ dans $\widehat{V}_{N}$ et pour tout $u_{1}, \ldots, \widehat{u}_{i}, \ldots, u_{n}$ et $v$ dans $\mathcal{O}_{N}$ nous ayons

$$
\max _{j}\left\{\left|\frac{u_{j}}{v}-y_{j}\right|\right\} \geqslant K|v|^{c} .
$$

Remarque 4. - En fait, comme le montre la preuve de cette proposition, deux cas peuvent se produire : soit aucun des polynômes $Q_{i}$ n'admet de solutions dans $\widehat{V}_{N}$, soit au moins un de ces polynômes en admet une. Dans le premier cas, la preuve nous permet de dire que la fonction de Artin de $P$ est bornée par une fonction de la forme $i \mapsto d i+c$ où $c \in \mathbb{N}$.

Dans le second cas, si la condition (1) est vérifiée, alors la fonction de Artin de $P$ est bornée par une fonction de la forme $i \mapsto d^{\prime} i+c$ où $c \in \mathbb{R}_{+}$et $d^{\prime}>d$ sont des constantes. 
Avant de donner la preuve de cette proposition, nous allons d'abord énoncer un lemme qui va nous permettre de reformuler le théorème de Artin dans le cas particulier des polynômes à zéro isolé :

LEMME 2.2. - Soit $P$ un polynôme de $\mathcal{O}_{N}\left[X_{1}, \ldots, X_{n}\right]$ ayant pour seul zéro à coordonnées dans $\mathcal{O}_{N}$ le point $(0, \ldots, 0)$. Le polynôme $P$ admet une fonction de Artin majorée par la fonction $\beta$, si et seulement si nous avons l'inégalité :

$$
\forall\left(x_{1}, \ldots, x_{n}\right) \in \mathcal{O}_{N}^{n}, \quad \operatorname{ord}\left(P\left(x_{1}, \ldots, x_{n}\right)\right) \leqslant \beta\left(\min _{k}\left\{\operatorname{ord}\left(x_{k}\right)\right\}\right) .
$$

En particulier $P$ admet une fonction de Artin majorée par une fonction affine, si et seulement si il existe deux constantes a et $b$ telles que

$$
\forall\left(x_{1}, \ldots, x_{n}\right) \in \mathcal{O}_{N}^{n}, \quad \operatorname{ord}\left(P\left(x_{1}, \ldots, x_{n}\right)\right) \leqslant a \min _{k}\left\{\operatorname{ord}\left(x_{k}\right)\right\}+b .
$$

Démonstration du lemme. - Supposons que la fonction de Artin de $P$ soit majorée par la fonction $\beta$. Soient $\left(x_{1}, \ldots, x_{n}\right) \in \mathcal{O}_{N}^{n}$ et $i \in \mathbb{N}$ tels que $P\left(x_{1}, \ldots, x_{n}\right) \in \mathfrak{m}^{\beta(i)+1}$ et $P\left(x_{1}, \ldots, x_{n}\right) \notin \mathfrak{m}^{\beta(i+1)+1}$. Alors d'après le théorème 1.1 , il existe $\left(\bar{x}_{1}, \ldots, \bar{x}_{n}\right) \in \mathcal{O}_{N}^{n}$ tel que pour tout $k, x_{k}-\bar{x}_{k} \in \mathfrak{m}^{i+1}$, et $P\left(\bar{x}_{1}, \ldots, \bar{x}_{n}\right)=0$. Par hypothèse sur $P$, nous avons nécessairement $\bar{x}_{k}=0$ pour tout $k$. Et donc $\operatorname{ord}\left(x_{k}\right) \geqslant i+1$. Nous avons donc l'inégalité (2).

Inversement si nous avons l'inégalité (2), et si $\left(x_{1}, \ldots, x_{n}\right) \in \mathcal{O}_{N}^{n}$ vérifie $P\left(x_{1}, \ldots, x_{n}\right) \in$ $\mathfrak{m}^{\beta(i)+1}$, alors nécessairement $x_{k} \in \mathfrak{m}^{i+1}$ pour tout $k$, et la fonction de Artin de $P$ est bornée par la fonction $\beta$.

Démonstration de la proposition. - Soient $P$ comme dans l'énoncé et $x_{1}, \ldots, x_{n} \in \mathcal{O}_{N}$. Quitte à changer le nom des variables, nous pouvons supposer que nous avons ord $\left(x_{1}\right) \leqslant \cdots \leqslant \operatorname{ord}\left(x_{n}\right)$. Nous avons alors

$$
P\left(x_{1}, \ldots, x_{n}\right)=x_{1}^{d} P\left(1, \frac{x_{2}}{x_{1}}, \ldots, \frac{x_{n}}{x_{1}}\right) .
$$

Notons alors $Q_{1}\left(Y_{2}, \ldots, Y_{n}\right)$ le polynôme $P\left(1, Y_{2}, \ldots, Y_{n}\right)$. Le polynôme $Q_{1}$ n'a pas de racine dans $\mathcal{O}_{N}$. Il n'en a donc pas non plus dans $V_{N}$, mais peut en avoir dans $\widehat{V}_{N}$.

Supposons que $Q_{1}$ n'ait pas de racine dans $\widehat{V}_{N}$. Comme $\widehat{V}_{N}$ est de la forme $\mathbb{K}[[T]]$ où $\mathbb{K}$ est un corps et $T$ une variable formelle, d'après le théorème de Greenberg (cf. [5]), $Q_{1}$ admet une fonction de Artin bornée par une constante $c$ et dans ce cas nous obtenons

$$
\operatorname{ord}\left(P\left(1, \frac{x_{2}}{x_{1}}, \ldots, \frac{x_{n}}{x_{1}}\right)\right)<c+1
$$

Donc

$$
\operatorname{ord}\left(P\left(x_{1}, \ldots, x_{n}\right)\right)<d \operatorname{ord}\left(x_{1}\right)+c+1 .
$$

Et donc, d'après le lemme 2.2, la fonction de Artin de $P$ est bornée par une fonction affine dont le coefficient de linéarité peut être choisi égal à $d$. Ceci correspond au premier cas énoncé dans la remarque 4.

Si $Q_{1}$ a des racines dans $\widehat{V}_{N}$, toujours d'après [5], $Q_{1}$ admet une fonction de Artin bornée par une fonction affine $i \mapsto \lambda i+\mu$. Notons $\left(y_{2}, \ldots, y_{n}\right)$ un plus proche zéro de $\left(\frac{x_{2}}{x_{1}}, \ldots, \frac{x_{n}}{x_{1}}\right)$ pour la topologie $\mathfrak{m}$-adique.

$4^{\text {e }}$ SÉRIE - TOME $38-2005-\mathrm{N}^{\circ} 6$ 
Supposons que nous ayons $\min _{j}\left\{\operatorname{ord}\left(\frac{x_{j}}{x_{1}}-y_{j}\right)\right\} \leqslant a \operatorname{ord}\left(x_{1}\right)+b$ où $a$ et $b$ sont des constantes. Alors nous avons

$$
\begin{aligned}
\operatorname{ord}\left(Q_{1}\left(\frac{x_{2}}{x_{1}}, \ldots, \frac{x_{n}}{x_{1}}\right)\right) & <\lambda\left(\min _{j}\left\{\operatorname{ord}\left(\frac{x_{j}}{x_{1}}-y_{j}\right)\right\}+1\right)+\mu+1 \\
& <\lambda\left(a \operatorname{ord}\left(x_{1}\right)+b+1\right)+\mu+1 .
\end{aligned}
$$

D'où

$$
\operatorname{ord}\left(P\left(x_{1}, \ldots, x_{n}\right)\right)<(a \lambda+d) \operatorname{ord}\left(x_{1}\right)+\lambda a(b+1)+\mu+1 .
$$

Et donc, là encore d'après le lemme 2.2, la fonction de Artin de $P$ est bornée par une fonction affine dont le coefficient de linéarité est strictement plus grand que $d$. Ceci correspond au second cas énoncé dans la remarque 4.

Inversement, supposons qu'il existe $i \in\{1, \ldots, n\}$ tel que, pour tout $c \in \mathbb{N}$, il existe une racine $\left(y_{1, c}, \ldots, \widehat{y}_{i, c}, \ldots, y_{n, c}\right)$ de $Q_{i}$ dans $\widehat{V}_{N}$ et des $u_{j, c}$ et $v_{c}$ dans $\mathcal{O}_{N}$ tels que

$$
\min _{j}\left\{\operatorname{ord}\left(\frac{u_{j, c}}{v_{c}}-y_{j, c}\right)\right\} \geqslant c \operatorname{ord}\left(v_{c}\right) .
$$

Comme $\operatorname{ord}\left(y_{j, c}\right) \geqslant 0$, nous avons $\operatorname{ord}\left(\frac{u_{j, c}}{v_{c}}\right) \geqslant 0$ pour tout $j$ et pour tout $c$ et donc $\min \left(\operatorname{ord}\left(v_{c}\right), \operatorname{ord}\left(u_{2, c}\right), \ldots, \operatorname{ord}\left(u_{n, c}\right)\right)=\operatorname{ord}\left(v_{c}\right)$.

Nous avons

$$
\begin{aligned}
Q_{i}\left(\frac{u_{1, c}}{v_{c}}, \ldots, \frac{u_{n, c}}{v_{c}}\right)= & Q_{i}\left(\frac{u_{1, c}}{v_{c}}, \ldots, \frac{u_{n, c}}{v_{c}}\right)-Q_{i}\left(y_{1, c}, \ldots, y_{n, c}\right) \\
= & Q_{i}\left(\frac{u_{1, c}}{v_{c}}, \ldots, \frac{u_{n, c}}{v_{c}}\right)-Q_{i}\left(y_{1, c}, \frac{u_{2, c}}{v_{c}}, \ldots, \frac{u_{n, c}}{v_{c}}\right) \\
& +Q_{i}\left(y_{1, c}, \frac{u_{2, c}}{v_{c}}, \ldots, \frac{u_{n, c}}{v_{c}}\right)-\cdots-Q_{i}\left(y_{1, c}, \ldots, y_{n, c}\right) .
\end{aligned}
$$

Or nous avons

$$
\begin{aligned}
& Q_{i}\left(y_{1, c}, \ldots, y_{j-1, c}, \frac{u_{j, c}}{v_{c}}, \ldots, \frac{u_{n, c}}{v_{c}}\right)-Q_{i}\left(y_{1, c}, \ldots, y_{j, c}, \frac{u_{j+1, c}}{v_{c}}, \ldots, \frac{u_{n, c}}{v_{c}}\right) \\
& \quad=\left(\frac{u_{j, c}}{v_{c}}-y_{j, c}\right) U_{i}
\end{aligned}
$$

où $U_{i} \in \widehat{V}_{N}$ (car $Q_{i}$ est à coefficients dans $\mathcal{O}_{N}$, et les $\frac{u_{j, c}}{v_{c}}$ et les $y_{j, c}$ sont dans $\widehat{V}_{N}$ ).

Nous en déduisons que

$$
\operatorname{ord}\left(Q_{i}\left(\frac{u_{2, c}}{v_{c}}, \ldots, \frac{u_{n, c}}{v_{c}}\right)\right) \geqslant \min _{j}\left\{\operatorname{ord}\left(\frac{u_{j, c}}{v_{c}}-y_{j, c}\right)\right\} \geqslant c \operatorname{ord}\left(v_{c}\right) .
$$

D'où

$$
\operatorname{ord}\left(P\left(v_{c}, u_{2, c}, \ldots, u_{n, c}\right)\right) \geqslant(c+d) \operatorname{ord}\left(v_{c}\right) .
$$

Donc $P$ n'admet pas de fonction de Artin bornée par une fonction affine, car $P$ ne vérifie pas l'inégalité (3) du lemme 2.2.

Nous déduisons de la proposition précédente le résultat d'approximation diophantienne suivant : 
Proposition 2.3. - Soit $x \in \widehat{\mathbb{K}}_{N}$ algébrique sur $\mathbb{K}_{N}$ tel que $x \notin \mathbb{K}_{N}$. Alors il existe une fonction $\gamma: \mathbb{N} \rightarrow \mathbb{N}$ telle que

$$
\operatorname{ord}\left(\frac{u}{v}-x\right) \leqslant \gamma(\operatorname{ord}(v))
$$

pour tous $u$ et $v$ dans $\mathcal{O}_{N}$.

Si $Q=a_{d} X^{d}+\cdots+a_{0}$ est le polynôme minimal de $x$ sur $\mathcal{O}_{N}$, alors $\gamma$ est majorée par une fonction affine si et seulement si la fonction de Artin de $P=a_{d} X^{d}+a_{d-1} X^{d-1} Y+\cdots+a_{0} Y^{d} \in$ $\mathcal{O}_{N}[X, Y]$ est majorée par une fonction affine, c'est-à-dire qu'il existe $a \geqslant 1$ et $K \geqslant 0$ tels que

$$
\left|\frac{u}{v}-x\right| \geqslant K|v|^{a}, \quad \forall u, v \in \mathcal{O}_{N} .
$$

Démonstration. - Soit $x \in \widehat{\mathbb{K}}_{N}$ algébrique sur $\mathbb{K}_{N}$. Si $\operatorname{ord}(x) \geqslant 0$, soit $Q$ un polynôme irréductible de $\mathcal{O}_{N}[X]$ tel que $Q(x)=0$ et $P(X, Y)$ l'homogénéisé de $Q$. Le corollaire découle alors de la proposition précédente si $P$ admet une fonction de Artin bornée par une fonction affine. Si ce n'est pas le cas, l'existence de la fonction de Artin de $P$, notée $\beta$, nous permet de dire, en reprenant l'inégalité (4) de la dernière partie de la preuve de la proposition précédente et grâce au lemme 2.2,

$$
\begin{aligned}
\beta(\operatorname{ord}(v)) & \geqslant \beta(\min \{\operatorname{ord}(u), \operatorname{ord}(v)\}) \geqslant \operatorname{ord}(P(u, v)) \\
& =\operatorname{ord}\left(Q\left(\frac{u}{v}\right)\right)+d \operatorname{ord}(v) \geqslant \operatorname{ord}\left(\frac{u}{v}-x\right)+d \operatorname{ord}(v) .
\end{aligned}
$$

Le résultat en découle directement.

Si ord $(x)<0$, notons $Q$ un polynôme irréductible de $\mathcal{O}_{N}[X]$ tel que $Q(x)=0$. Soit $P(X, Y)$ l'homogénéisé de $Q$ et $R(Y)=P(1, Y)$. Le polynôme $R$ est irréductible, de même degré que $Q$ et $R(1 / x)=0$. Nous avons alors $Q\left(\frac{u}{v}\right)=\frac{u^{d}}{v^{d}} R\left(\frac{v}{u}\right)$. Si le terme initial de $\frac{u}{v}$ est différent du terme initial de $x$ pour la valuation ord, alors nous obtenons ord $\left(\frac{u}{v}-x\right) \leqslant \operatorname{ord}(x)$. Sinon, $\operatorname{ord}(u)+\operatorname{ord}(x)=\operatorname{ord}(v)$. Sachant que $1 / x \in \widehat{V}_{N}$, d'après le lemme 2.2 et l'existence de la fonction de Artin $\beta$ du polynôme $P$, nous obtenons comme précédemment l'inégalité $\operatorname{ord}\left(\frac{v}{u}-\frac{1}{x}\right) \leqslant \beta(\operatorname{ord}(u))-d \operatorname{ord}(u) \leqslant \beta(\operatorname{ord}(u))$.

Donc nous avons

$$
\begin{aligned}
\operatorname{ord}\left(\frac{u}{v}-x\right) & =\operatorname{ord}\left(\frac{u x}{v}\left(\frac{v}{u}-\frac{1}{x}\right)\right) \\
& =\operatorname{ord}\left(\frac{v}{u}-\frac{1}{x}\right) \leqslant \beta(\operatorname{ord}(v)-\operatorname{ord}(x)) .
\end{aligned}
$$

Dans tous les cas, nous avons ord $\left(\frac{u}{v}-x\right) \leqslant \beta(\operatorname{ord}(v)-\operatorname{ord}(x))+\operatorname{ord}(x)$.

Exemple 2.4. - Supposons que la caractéristique de $\mathbb{k}$ est différente de 2. Nous présentons ici une suite $\left(x_{p}\right)_{p \in \mathbb{N} \backslash\{0,1,2\}}$ d'éléments de $\widehat{\mathbb{K}}_{N}$, chacun de degré 2 sur $\mathbb{K}_{N}$, pour lesquels il existe $u_{p, k}$ et $v_{p, k}$ tels que $\left|x_{p}-\frac{u_{k, p}}{v_{k, p}}\right|=C_{p}\left|v_{k}\right|^{\frac{p}{2}-1}$ et $\operatorname{ord}\left(v_{p, k}\right)$ tend vers $+\infty$ avec $k$, où $C_{p}$ est une constante qui dépend de $p$. Nous voyons donc que s'il existe une version linéaire d'approximation diophantienne pour les séries en plusieurs variables, contrairement au cas des nombres réels algébriques, la meilleure borne $c$ telle qu'il existe $K$ avec

$$
\left|x-\frac{u}{v}\right|>K|v|^{c}, \quad \forall u, v \in \mathcal{O}_{N}
$$

$4^{\mathrm{e}}$ SÉRIE - TOME $38-2005-\mathrm{N}^{\circ} 6$ 
ne peut pas être bornée par le degré de l'extension de $\mathbb{K}_{N}$ par $x$. Il n'existe donc pas de version du théorème de Liouville pour les extensions finies de $\mathbb{K}_{N}$ dans $\widehat{\mathbb{K}}_{N}$.

Soit $P_{p}(X, Y)$ le polynôme $X^{2}-\left(T_{1}^{2}+T_{2}^{p}\right) Y^{2}$ avec $p \in \mathbb{N} \backslash\{0,12\}$. Le terme $T_{1}^{2}+T_{2}^{p}$ n'est pas un carré car la caractéristique du corps de base est différente de 2 . Le seul zéro de $P_{p}$ est alors $(0,0)$. Soit $Q_{p}(X)=X^{2}-\left(T_{1}^{2}+T_{2}^{p}\right)$. Le polynôme $Q_{p}$ a deux racines dans $\widehat{V}_{N}$ qui sont $x_{p}$ et $-x_{p}$ avec

$$
x_{p}=T_{1}\left(1+\frac{1}{2} \frac{T_{2}^{p}}{T_{1}^{2}}-\frac{1}{8} \frac{T_{2}^{2 p}}{T_{1}^{4}}+\cdots+\frac{(-1)^{n-1}(2 n-2) !}{2^{2 n-1}(n-1) ! n !} \frac{T_{2}^{n p}}{T_{1}^{2 n}}+\cdots\right) .
$$

Notons $a_{n}:=(-1)^{n-1} \frac{(2 n-2) !}{2^{2 n-1}(n-1) ! n !}$. Soit $k$ un entier positif ; notons

$$
x_{p, k}:=T_{1} \sum_{i=0}^{k-1} a_{i} \frac{T_{2}^{i p}}{T_{1}^{2 i}}
$$

Nous avons

$$
x_{p}-x_{p, k}=T_{1} \sum_{i \geqslant k} a_{i} \frac{T_{2}^{i p}}{T_{1}^{2 i}} \in \widehat{\mathfrak{m}}_{N}^{(p-2) k+1}
$$

et $x_{p, k}$ s'écrit sous la forme $\frac{u_{p, k}}{v_{p, k}}$ avec $u_{p, k}$ et $v_{p, k}$ premiers entre eux et $v_{p, k}=T_{1}^{2 k-3}$. D'où

$$
\begin{gathered}
\operatorname{ord}\left(x_{p}-\frac{u_{p, k}}{v_{p, k}}\right)=k(p-2)+1=\left(\frac{p}{2}-1\right) \operatorname{ord}\left(v_{p, k}\right)+\frac{3}{2} p-2, \\
\text { ou encore }\left|x_{p}-\frac{u_{p, k}}{v_{p, k}}\right|=e^{-\frac{3}{2} p+2}\left|v_{p, k}\right|^{\frac{p}{2}-1} .
\end{gathered}
$$

Exemple 2.5. - Plus généralement, S. Izumi a étudié les polynômes de la forme $X^{d}-a Y^{d}$ où $a$ n'est pas une puissance $d$-ième dans $\mathcal{O}_{N}$, et a montré que la fonction de Artin de ces polynômes est bornée par une fonction affine (cf. [8, proposition 5.1]), sans pour autant donner de bornes explicites.

\section{Preuve du théorème 1.2}

Nous pouvons donner la preuve du théorème 1.2 annoncé dans l'introduction. Celle-ci consiste à interpréter différemment le contre-exemple 2.4 à l'approximation diophantienne de type Liouville :

Soit $N \geqslant 2$ fixé. Nous notons $P(X, Y, Z):=X^{2}-Y^{2} Z$. Supposons la caractéristique de $\mathbb{k}$ différente de 2 . Soient $p$ et $k$ des entiers strictement plus grands que 2 et soient

$$
u_{p, k}=T_{1}^{2 k-2} \sum_{i=0}^{k-1} a_{i} \frac{T_{2}^{i p}}{T_{1}^{2 i}}, \quad v_{k}=T_{1}^{2 k-3}, \quad \text { et } \quad z_{p}=T_{1}^{2}+T_{2}^{p}
$$

avec, pour tout $n \in \mathbb{N}, a_{n}:=(-1)^{n-1} \frac{(2 n-2) !}{2^{2 n-1}(n-1) ! n !}$.

Notons, comme dans l'exemple 2.4, $x_{p, k}:=\frac{u_{p, k}}{v_{k}}=T_{1} \sum_{i=0}^{k-1} a_{i} \frac{T_{2}^{i p}}{T_{1}^{2 i}}$, et $x_{p}:=T_{1} \sum_{i \geqslant 0} a_{i} \frac{T_{2}^{i p}}{T_{1}^{2 i}}$. En particulier $x_{p}^{2}=z_{p}$. 
Alors

$$
\begin{aligned}
P\left(u_{p, k}, v_{k}, z_{p}\right) & =\left(T_{1}^{2}\left(\sum_{i=0}^{k-1} a_{i} \frac{T_{2}^{i p}}{T_{1}^{2 i}}\right)^{2}-\left(T_{1}^{2}+T_{2}^{p}\right)\right) T_{1}^{4 k-6} \\
& =\left(\left(\frac{u_{p, k}}{v_{k}}\right)^{2}-z_{p}\right) v_{k}^{2} \\
& =\left(x_{p, k}-x_{p}\right)\left(x_{p, k}+x_{p}\right) v_{k}^{2} \in \widehat{\mathfrak{m}}_{N}^{(p+2) k-4}
\end{aligned}
$$

d'après la relation $(5)$ de l'exemple 2.4.

$\mathrm{Si}(x, y, z)$ est un zéro de $P$ alors soit $z$ est un carré, soit $x=y=0$. Or

$$
\sup _{t \in \mathcal{O}_{N}}\left(\operatorname{ord}\left(z_{p}-t^{2}\right)\right)=p
$$

car la caractéristique de $\mathbb{k}$ est différente de 2 , et

$$
\min \left(\operatorname{ord}\left(u_{p, k}\right), \operatorname{ord}\left(v_{k}\right)\right)=2 k-3 .
$$

Donc, en posant $p=k-2$, nous avons

$$
P\left(u_{k-2, k}, v_{k}, z_{k-2}\right) \in \mathfrak{m}^{k^{2}-4}
$$

et

$$
\sup \left(\min \left\{\operatorname{ord}\left(u_{k-2, k}-x\right), \operatorname{ord}\left(v_{k}-y\right), \operatorname{ord}\left(z_{k-2}-z\right)\right\}\right) \leqslant 2 k-3
$$

où la borne supérieure est prise sur toutes les racines $(x, y, z)$ de $P$.

Notons $\beta_{N}$ la fonction de Artin de $P$. Soient $i \in \mathbb{N}^{*}$ pair et $k \in \mathbb{N}$ tel que $i=2 k-2$. D'après ce qui précède, il existe une solution approchée de $P$ à l'ordre $\left(\frac{i+2}{2}\right)^{2}-4=\left(\frac{i+2}{2} t\right)^{2}-5+1$, mais il n'existe aucune solution de $P$ «proche» de cette solution à l'ordre $i+1$. Donc $\beta_{N}(i) \geqslant$ $\left(\frac{i+2}{2}\right)^{2}-5$. Si $i$ est impair, comme $\beta_{N}(i) \geqslant \beta_{N}(i-1)$, nous avons $\beta_{N}(i) \geqslant\left(\frac{i}{2}\right)^{2}-5$. Donc pour tout $i \in \mathbb{N}^{*}$, nous avons

$$
\beta_{N}(i) \geqslant \frac{i^{2}}{4}-5
$$

\section{Application : non-existence d'élimination des quantificateurs dans le corps $\mathbb{k}\left(\left(T_{1}, \ldots, T_{N}\right)\right)$ pour $N \geqslant 2$}

J. Denef et F. Loeser [4] ont donné une preuve du théorème de Greenberg à l'aide d'un résultat d'élimination des quantificateurs dû à J. Pas [10]. Nous pouvons appliquer ici la même méthode pour montrer que dans le cas $N \geqslant 2$, il n'y a pas d'existence d'une théorie d'élimination des quantificateurs dans le corps $\mathbb{k}\left(\left(T_{1}, \ldots, T_{N}\right)\right)$ muni du langage défini ci-dessous.

Dans cette partie, $\mathbb{k}$ est un corps algébriquement clos.

Soit $\mathcal{L}_{\text {Pre }}$ le langage du premier ordre dont les variables sont les éléments de $\mathbb{Z}$ et les symboles sont $+, \leqslant, 0,1$ et pour tout $d \in \mathbb{Z}_{\geqslant 2}$ un symbole pour signifier la relation binaire $x \equiv y \bmod d$.

Soit $\mathcal{L}_{\mathbb{k}}$ le langage du premier ordre dont les variables sont les éléments de $\mathbb{k}$ et les symboles sont $+,-, \times, 0,1$.

Soit $\mathcal{L}_{N}$ le langage du premier ordre dont les variables sont les éléments de $\mathbb{K}_{N}$ et les symboles sont $+,-, \times, 0,1$ et le symbole pour la fonction ord.

Nous pouvons alors énoncer le résultat suivant dû à F. Delon [3] :

$4^{\text {e }}$ SÉRIE - TOME $38-2005-\mathrm{N}^{\circ} 6$ 
THÉORÈme 4.1. - Soit $N \geqslant 2$ fixé. Considérons le langage du premier ordre à trois sortes $\mathcal{L}:=\left(\mathcal{L}_{\text {Pre }}, \mathcal{L}_{\mathbb{k}}, \mathcal{L}_{N}\right.$, ord,$\left.\pi\right)$, où $\pi$ est une fonction de $\mathbb{K}_{N}$ vers $\mathbb{k}$. Soit $\mathcal{L}^{\prime}$ le langage formé à partir de $\mathcal{L}$ en lui ajoutant autant de symboles que l'on veut de telle sorte que la restriction de $\mathcal{L}^{\prime}$ à $\mathbb{Z}$ soit égale à $\mathcal{L}_{\text {Pre. }}$. Alors $\mathcal{L}^{\prime}$ n'admet pas d'élimination des $\mathbb{K}_{N}$-quantificateurs.

Démonstration. - Considérons $\beta$, la fonction de Artin du polynôme $P=X^{2}-Z Y^{2}$ vu comme polynôme de $\mathcal{O}_{N}[X, Y, Z]$. Cette fonction peut se définir dans les langages $\mathcal{L}$ et $\mathcal{L}^{\prime}$, et donc son graphe aussi. En effet, pour tout entier $n$ nous avons

$$
\begin{aligned}
\left(P(x, y, z) \in \mathfrak{m}^{\beta(n)+1} \Longrightarrow\right. & \left(\exists \bar{x}, \bar{y}, \bar{z} /\left(x-\bar{x}, y-\bar{y}, z-\bar{z} \in \mathfrak{m}^{n+1}\right)\right. \\
& \wedge P(\bar{x}, \bar{y}, \bar{z})=0)), \\
\wedge\left(P(x, y, z) \in \mathfrak{m}^{\beta(n)} \Longrightarrow\right. & \left(\neg \exists \bar{x}, \bar{y}, \bar{z} /\left(x-\bar{x}, y-\bar{y}, z-\bar{z} \in \mathfrak{m}^{n+1}\right)\right. \\
& \wedge P(\bar{x}, \bar{y}, \bar{z})=0)) .
\end{aligned}
$$

Si $\mathcal{L}^{\prime}$ admettait une élimination des $\mathbb{K}_{N}$-quantificateurs, alors d'après un résultat de Presburger, qui dit que $\mathcal{L}_{\text {Pre }}$ admet une élimination des quantificateurs (cf. [11]), et d'après le théorème de constructibilité de Chevalley, le langage $\mathcal{L}^{\prime}$ admettrait une élimination des quantificateurs. Le graphe de $\beta$, inclus dans $\mathbb{Z}^{2}$, serait donc semi-algébrique dans le langage $\mathcal{L}_{\text {Pre }}$ et il existerait alors une partition finie de $\mathbb{N}$ en classes de congruences telle que $\beta$ soit affine sur chacune de ces classes, ce qui est faux d'après ce qui précède.

Remarque 5. - Pour $N=1$ un résultat d'élimination des quantificateurs a été obtenu par J. Pas (cf. [10]).

\section{Remerciements}

Je suis très reconnaissant à $\mathrm{M}$. Hickel et $\mathrm{M}$. Spivakovsky pour leurs conseils et encouragements. Je tiens aussi à remercier J. Nicaise pour m'avoir fait remarquer que l'élimination des quantificateurs dans $\mathbb{K}_{N}$ impliquait que toute fonction de Artin était bornée par une fonction affine.

\section{RÉFÉRENCES}

[1] ARTIN M., Algebraic approximation of structures over complete local rings, Publ. Math. IHÉS 36 (1969) 23-58.

[2] Delfino D., SWANSON I., Integral closure of ideals in excellent local rings, J. Algebra 187 (1997) 422-445.

[3] DELON F., Indécidabilité de la théorie des anneaux de séries formelles à plusieurs indéterminées, Fund. Math. 112 (1981) 215-229.

[4] DeneF J., LOESER F., Germs of arcs on singular algebraic varieties and motivic integration, Invent. Math. 135 (1999) 201-232.

[5] Greenberg M.J., Rational points in henselian discrete valuation rings, Publ. Math. IHÉS 31 (1966) 59-64.

[6] HiCKEL M., Fonction de Artin et germes de courbes tracées sur un germe d'espace analytique, Amer. J. Math. 115 (1993) 1299-1334.

[7] Hickel M., Calcul de la fonction d'Artin-Greenberg d'une branche plane, Pacific J. Math. 213 (2004) 37-47.

[8] IzUMi S., A measure of integrity for local analytic algebras, Publ. RIMS Kyoto Univ. 21 (1985) 719736.

[9] LeJeune-JALABert M., Courbes tracées sur un germe d'hypersurface, Amer. J. Math. 112 (1990). 
[10] PAS J., Uniform $p$-adic cell decomposition and local zeta functions, J. reine angew. Math. 399 (1989) $137-172$.

[11] Presburger M., Über die Vollständigkeit eines gewissen Systems des Arithmetik ganzer Zahlen, im welchem die Addition als einzige Operation hervortritt, Comptes-Rendus du Premier Congrès des Mathématiciens des Pays Slaves 395 (1927) 92-101.

[12] Rond G., Lemme d'Artin-Rees, théorème d'Izumi et fonctions de Artin, J. Algebra (2006), à paraître.

[13] SPIVAKOVSKY M., Valuations, the linear Artin approximation theorem and convergence of formal functions, in : Proceedings of the II SBWAG, in : Alxebra, vol. 54, 1990, pp. 237-254.

[14] WAVRIK J.J., A theorem on solutions of analytic equations with applications to deformations of complex structures, Math. Ann. 216 (1975) 127-142.

(Manuscrit reçu le 9 septembre 2005; accepté, après révision, le 18 octobre 2005.)

Guillaume ROND

Department of Mathematics,

University of Toronto,

Toronto, Ontario M5S 2E4,

Canada

E-mail : rond@picard.ups-tlse.fr 